\title{
Contact angle measurements with constant drop volume. Control of wettability of some materials by physico-chemical treatment Vladimir I. Bredikhin ${ }^{1 *}$, Nikita M. Bityurin ${ }^{2}$
}

Institute of Applied Physics RAS, Nizhny Novgorod, Russia

Email: bredikh@appl.sci-nnov.ru (*corresponding author)

\begin{abstract}
A modified express method of contact angle measurement based on determining the diameter of a drop with a known volume has been elaborated. The method offers a number of advantages: it can be used in an express manner for in situ dynamic investigation and makes it possible to observe inhomogeneity and anisotropy of hydrophilicity. The method is convenient in comparative measurements and does not require any special equipment.

Using the proposed method, the possibility of hydrophilic control of some materials (silicate glass, TiO ${ }_{2}$ (anataze) and polymetilmetacrylate (PMMA)) by UV (ultra violet) and chloroform vapor treatment has been studied. In particular, hydrophilicity of PMMA after UV irradiation was observed, and it is shown that changes in hydrophilicity are connected with the surface oxidation of PMMA. The observed phenomenon of hydrophilicity control can be used in various technologies, such as liquid coating, printing, spray quenching, nanolithography, pharmacology and others.
\end{abstract}

Keywords - Contact angle, constant drop volume, control of hydrophilicity, physico-chemical treatment, PMMA, inorganic glass, $\mathrm{TiO}_{2}$.

\section{INTRODUCTION}

Wetting between different liquids and solids have received tremendous interest from both fundamental and applied points of view. It plays an important role in many chemical technologies, such as oil recovery, lubrication, liquid coating, printing, spray quenching, and lithography [1, 2]. In recent years, there has been an increasing interest in the study of superhydrophobic and superhydrophilic surfaces, due to their potential applications in, for example, self-cleaning, nanofluidics, electrowetting, and nanolithography. Traditional way to study wetting is quantitative measurement of contact angle $\theta$, which characterizes the shape of a liquid drop on a solid surface (or on another liquid) (see Fig. 1) [1, 2].

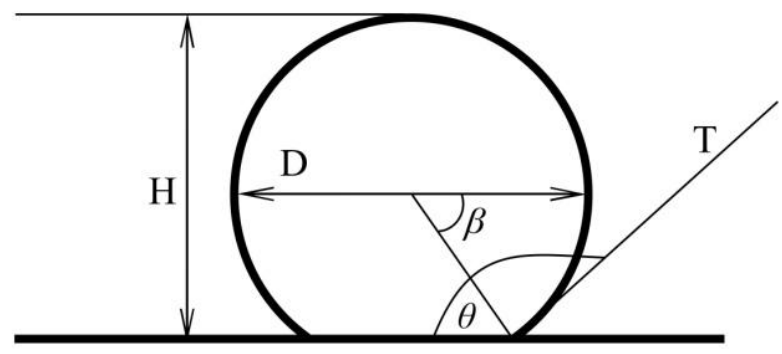

FIG. 1. DROP ON A SOLID SURFACE.

Currently, the primary method of measuring the contact angle $\theta$ is to observe the shape of the drop (volume of the order of 1 $\mu \mathrm{cL}$ ) and measure the angle between the drop tangent $\mathrm{T}$ and the solid surface at the drop-solid contact point either directly or by calculating it from the drop diameter D and height $\mathrm{H}$. Several methods and apparatuses were developed which permit to investigate wetting over the whole range of contact angles $0^{\circ}<\theta<180^{\circ}$ in static and in dynamic regimes. Nevertheless, these methods have some limitations: they are not fast enough, do not allow investigating the anisotropy and homogeneity of wetting, and require special equipment.

\section{CONTACT ANGLE MEASUREMENT FROM A TOP VIEW IMAGE OF A DROP AT KNOWN DROP VOLUME}

In Fig. 1 it is evident that knowing the drop diameter D only is not enough to calculate the angle $\theta$. For this purpose, it is convenient to use the drop volume $V$. In Fig. 1 it can be clearly seen that the volume $V$ and the drop radius $R$ (in the spherical drop approximation) are related to the angle $\theta$ by

$$
V=\pi R^{3}\left|\int_{\pi-\theta}^{0}(\sin \beta)^{3} d \beta\right|
$$




$$
V=\pi R^{3}\left(\frac{2}{3}+\left(\frac{\cos \theta^{3}}{3}\right)-\cos \theta\right)
$$

Expression (1a) gives limit cases:

$$
\begin{array}{lll}
\theta \rightarrow \pi & R \rightarrow(3 / 4 \pi)^{1 / 3} V^{1 / 3} & \text { (superhydrophobic case) } \\
\theta \rightarrow 0 & R \rightarrow \infty & \text { (superhydrophilic case) }
\end{array}
$$

\section{DROPS OF KNOWN VOLUME}

From Eqs. (1) and (1a) it can be seen that it is possible to compare wetting for different measurements immediately if the volumes of drops are the same: larger diameter means better wetting and smaller angle $\theta$. Therefore, this method is suitable for rapid, comparative investigations. For quantitative measurements of the contact angle $\theta$ by analyzing the drop shape from above one has to know the drop volume $V$ and then calculate $\theta$ from Eq. 1a. It is useful to have drops of constant volume. To produce such drops, different micro dozers can be used, in particular, micro dozers based on capillary. In our experiments, the results of which will be discussed below, we used a standard syringe $(\sim 1 \mathrm{~mL})$ with a normally clipped needle tip. The needle played the role of a micro-capillary when it was immersed in water (without additional piston force). Then the drop was pushed carefully from the capillary to a substrate by the piston. By using such a simple technique and maintaining stable operation conditions, water drops of $\sim 1.5 \mu \mathrm{cL}$ stable volume were produced.

The value of the drop volume $V$ can be determined by measuring the volume of a sum of drops or alternatively by using the method of comparison, i.e. by fixing the drop on a substrate with a known contact angle $\theta$ known and calculating $V$ from Eq (1a). Fig. 2 shows an example of the dependence of contact angle $\theta$ on drop radius on logarithmic scales when the drop volume is $1 \mu \mathrm{cL}$. Note that the comparison method is very effective for express investigation of different substrates or in the process of treatment of the substrate or the liquid.

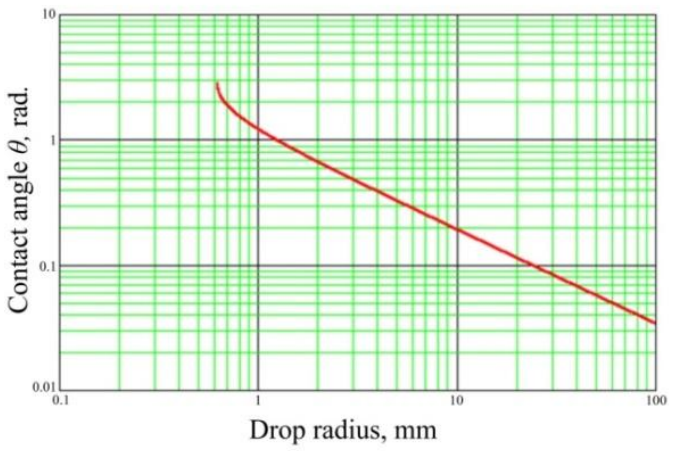

\section{FIG. 2. DEPENDENCE OF CONTACT ANGLE $\boldsymbol{\theta}$ ON DROP RADIUS. DROP VOLUME IS $1 \mu \mathrm{cL}$}

\section{EXPERIMENTS ON WETTING CONTROL IN SEVERAL MATERIALS}

\subsection{Comparative study of the effect of UV irradiation on wetting of selected materials}

We studied the impact of ultra violet UV irradiation (UV adaptation, UVA) on wettability of some transparent materials: silicate glass, polymetilmetacrylate (PMMA) and $\sim 0.1 \mu \mathrm{m} \mathrm{TiO}_{2}$ film (anataze) deposited on an object plate by the sol-gel method in a centrifuge $[3,4]$. In our experiments we used the "classic" conventional method of contact angle measurement, in which the drop shape is measured laterally, and the above-discussed modified method, in which areas of drops of the same volume are compared. We compared the shape and transverse size of a water drop on a substrate surface (i.e. top view of the drop) before and after treatment of the surface. The schematic diagram of the experiments is presented in Fig. 3.

The substrate surface was irradiated by a mercury-discharge lamp PRK-4 (Russia, (nominal power of optical radiation $220 \mathrm{~W}$, spectral UV range 180-400 $\mathrm{nm}$ ) with exposure time up to $40 \mathrm{~min}$ for the distance between the lamp and the substrate $\sim 20 \mathrm{~cm}$, half of the substrate being shielded. The samples were illuminated by a full-spectrum light of the lamp, as well as by sections of the spectrum isolated by color filters. After irradiation, several water drops of the same volume $\left(\sim 1.5 \cdot 10^{-3} \mu \mathrm{cL}\right)$ on the substrate were recorded by a digital photo camera. Similar measurements were made using the conventional method.

The bulk of experiments were done using the fixed drop volume method as it appeared to fit well the purpose of our study. This method is an express one, enables to directly compare results of surface treatment, makes it possible to visually see the non-uniformity and anisotropy of wetting, requires no unique equipment and can be used in any laboratory. Results of the 
series of experiments revealed that hydrophilicity increased substantially (fig. 4) under illumination by full-spectrum light for all the three materials. Wettability of the PMMA exposed to UV radiation became comparable to the wettability of glass, and its contact angle changed due to treatment from $\sim 60^{\circ}$ to $\sim 35^{\circ}$.

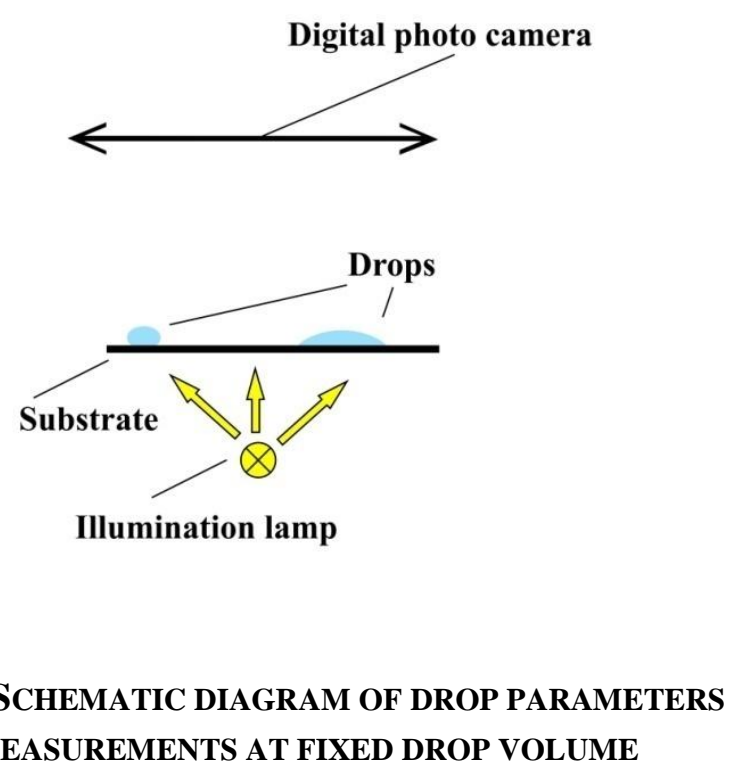

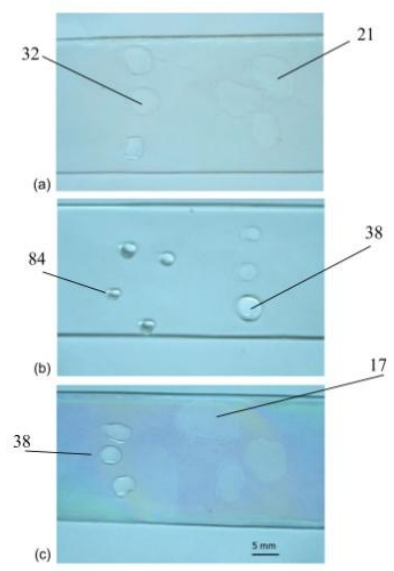

FIG. 4. WATER DROPS ON SILICATE GLASS (A),

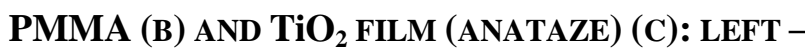
BEFORE UV IRRADIATION, RIGHT - AFTER UV IRRADIATION. NUMBERS SHOW CONTACT ANGLE IN DEG. CALCULATED FROM EQ. (1a)

Note that the UV-exposed $\mathrm{TiO}_{2}$ film wettability increased most of all (superhydrophilicity $[3,4]$ ), and characterization of wetting by the contact angle only is not enough. The diameter variation and asymmetry of drops (especially at large hydrophilicity) are seen in Figs. 4a and 4c, indicating inhomogeneity and anisotropy of wetting.

A more detailed study of PMMA hydrophilicity with isolation of dedicated spectral sections, more exactly, when the shortwave section shorter than $240 \mathrm{~nm}$ was cut off, did not reveal any significant increase of wettability. This fact shows that the radiation at the wavelength less than $240 \mathrm{~nm}$ influences PMMA hydrophilicity only.

\subsection{Comparative study of the effect of UV irradiation on PMMA wetting by different methods}

Results of the experiments on measuring the dependence of PMMA wettability contact angle on the time of exposure to fullspectrum lamp PRK 4 using the conventional_method are shown in fig. 5 for two distances (11 and $21 \mathrm{~cm})$ from the sample to the lamp. The contact angle was measured conventionally by making lateral photos of a drop having volume $\sim 1.5 \cdot 10^{-3} \mathrm{~mL}$ by a digital photo camera. Each point in fig. 5 corresponds to an individual sample averaged by 5 drops. Comparing data Fig.5 and Fig. 4 for PMMA it is seen that the modified and "classic" methods give practically the same results.

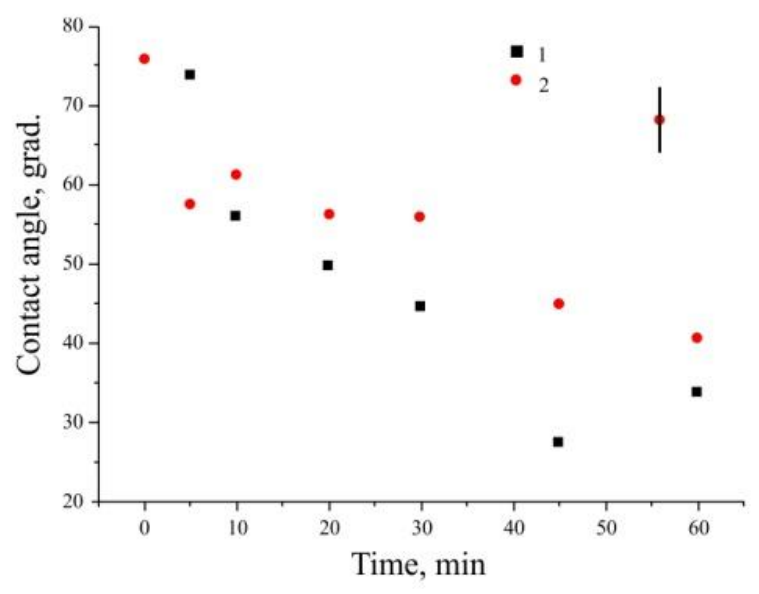

Fig. 5. DEPENDENCE OF CONTACT ANGLE OF $\mathrm{H}_{2} \mathrm{O}$ ON PMMA ON UV EXPOSURE TIME. 1 AND 2 - DISTANCE BETWEEN LAMP AND SUBSTRATE WAS $11 \mathrm{~cm}$ AND $21 \mathrm{~cm}$, RESPECTIVELY. 


\subsection{The effect of UV laser irradiation on PMMA wetting}

To clear the question about the nature of UV hydrophilization of PMMA, we have carried out a comparative study of PMMA

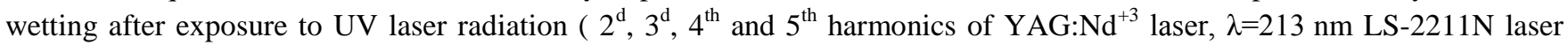
operating at a zero-order single transverse mode and with the 5th harmonic converter HG5) in laboratory and argon atmosphere. Wettability was investigated, as described earlier, by the modified method, using fixed drop volume. Irradiation was performed in a flow-through gas (Ar) cell with hermetic optical quartz windows. During irradiation in laboratory atmosphere, Ar was shut off and the windows were opened slightly to retain the irradiation dose.

The irradiation by the $2^{\mathrm{d}}, 3^{\mathrm{d}}$ and $4^{\text {th }}$ harmonics had no effect on PMMA wetting. The $5^{\text {th }}$ harmonic irradiation showed wetting enhancement only. The results of the series of experiments demonstrate that the drops placed on irradiated areas in Ar atmosphere do not spread, in contrast to the drop placed in the area irradiated in the presence of oxygen (Fig. 6). It means that the wettability rises under UV irradiation in the presence of oxygen only.

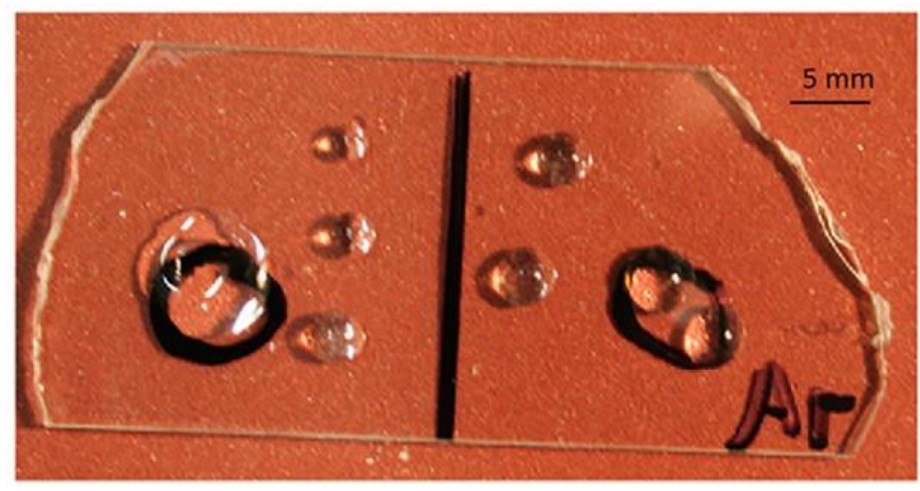

FIG. 6. TYPICAL SHAPE OF WATER DROPS ON UV IRRADIATED PMMA SURFACE. THE IRRADIATED AREA IS ENCIRCLED: LEFT - LABORATORY ATMOSPHERE, RIGHT - ARGON ATMOSPHERE. AVERAGE POWER OF UV IRRADIATION $20 \mathrm{~mW} / \mathrm{cm}^{2}$, IRRADIATION DOSE $\sim 4.8 \mathrm{~J} / \mathrm{cm}^{2}$.

\section{WETTABILITY OF PMMA AFTER TREATMENT WITH CHLOROFORM VAPOR}

In the literature, the processing of PMMA surfaces in chloroform vapor, which is a strong solvent for PMMA, is known [5, 6]. With this treatment, "smoothing" of the surface with a noticeable decrease in micro-roughness was observed. It is useful to investigate the effect of such treatment on the hydrophilicity of the surface. The scheme of the experiments carried out for this purpose is shown in Fig. 7. The PMMA substrate was placed on a chemical beaker with chloroform, so that only the central part of the substrate overlapped. The temperature of chloroform is $\sim 30^{\circ} \mathrm{C}$. The holding time is about a minute. Then, drops of water were applied to the areas treated and untreated in pairs, which, as before, were photographed. An example of the resulting picture is shown in Fig. 8. As in the case of UV treatment, it is evident that the droplet diameter on the treated surface is on average much larger than on the untreated surface. This indicates greater wettability on the treated part. The spread of diameters, apparently, is the result of surface wettability inhomogeneity, appeared after vaporization
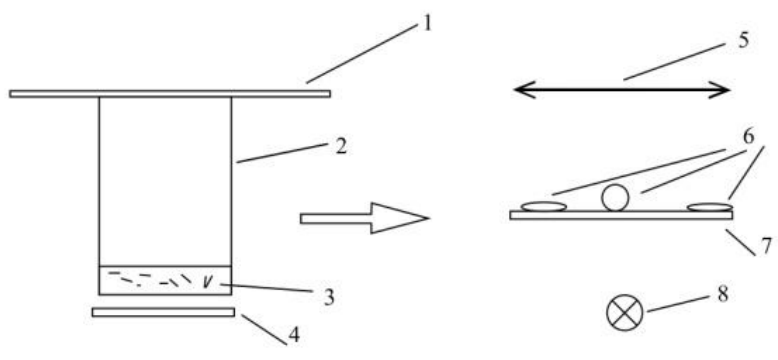

FIG. 7. SCHEME OF THE STUDY OF THE EFFECT OF CHLOROFORM VAPOR ON THE HYDROPHILICITY OF SAMPLES. 1-PMMA PLATE, 2-BEAKER, 3CHLOROFORM, 4- HEATER; 5-CAMERA, 6 -DROPS, 7 ILLUMINATOR

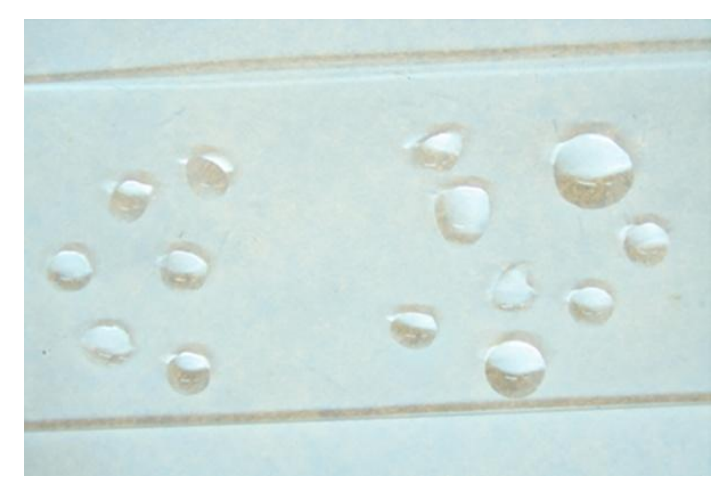

FIG. 8. WATER DROPLETS ON PMMA AFTER TREATMENT IN CHLOROFORM VAPOR. LEFT UNTREATED AREA， RIGHT - PROCESSED 


\section{DISCUSSION AND RESULTS}

For investigation of the influence of UV treatment on hydrophilicity of some materials, we used the modified method of measuring the contact angle of a drop on surface that had been specially elaborated by us. Measurements were made by determining the diameter of the drop of known volume from top-view images. The measurements were done, as a rule, in express, comparative regime to maintain equal conditions for drops applied on substrate parts prepared in an individual manner. To compare the methods, investigations were also performed for PMMA using the conventional method of measuring the contact angle from a side-view image of the drop. Results of the comparison show that the modified method permits to obtain the same results in an express manner using apparatuses of common use only. Moreover, the modified method is able to provide additional information, for example, on anisotropy, homogeneity and kinetics of wetting. The results obtained by this method may be significantly more important in some cases, in particular, for the case of superhydrophilicity, when the value of drop surface is more informative than the contact angle. Note, in case of $\mathrm{TiO}_{2}(\mathrm{Fig}$. 4c) the height of the drop is $\sim 10 \mu \mathrm{cm}$, and the conventional measurement of contact angle is not effective.

During comparison of the methods, new information on the possibility of UV PMMA hydrophilization and its nature was obtained. It was shown in experiment that a PMMA surface may be hydrophilized significantly by exposure to UV radiation. It was also demonstrated that the section of the spectrum shorter than $240 \mathrm{~nm}$ is responsible for the increase of wettability, in particular, the 5th harmonic irradiation is effective for PMMA wetting. It was shown that UVA is connected with oxygen.

It is well known [7] that photo-oxidation of polymers results in formation of multiple hydroxyl groups. The establishment of hydrogen bonds between these groups within the superficial layers of polymer and the water molecules would lead to wettability enhancement. The significance of photo-oxidation in the process of photodestruction of PMMA irradiated by the fifth harmonic of a neodymium laser was demonstrated experimentally in [8]. Therefore we explain the increase in PMMA wettability due to UVA by surface photo-oxidation.

The information on PMMA UVA is of interest for nanosphere lithography $[9,10]$ especially.

Additionally, the possibility of PMMA hydrophylization in chloroform vapor was shown. This effect can be connected with decomposition and volatilization of surface organic contaminations [11].

All presented quantitative results on contact angle were obtained in the spherical drop approximation (as the majority of results in the literature $[1,2])$. If greater precision is needed, the modified method can be generalized to account for the effect of, for example, gravity on the drop shape [2,12].

\section{CONCLUSION}

A modified express method of contact angle measurement based on determining the diameter of a drop with a known volume has been elaborated. The method offers a number of advantages: it can be used in an express manner for in situ dynamic investigation and makes it possible to observe inhomogeneity and anisotropy of hydrophilicity. The method is convenient in comparative measurements and does not require any special equipment.

Using the proposed method, the possibility of hydrophilic control of some materials (silicate glass, $\mathrm{TiO}_{2}$ (anataze) and PMMA) by UV and chloroform vapor treatment has been studied. In particular, it is shown that the change in PMMA hydrophilicity during UVA process is connected with the surface oxidation of PMMA. The revealed possibility of hydrophilicity control can be used in various technologies, such as liquid coating, printing, spray quenching, nanolithography, pharmacology and others.

\section{ACKNOWLEDGEMENTS}

This work was funded by the Russian Federal Agency for Scientific Organizations as a part of the State Assignment for the Institute of Applied Physics RAS under Project No. 0035-2014-0019.

\section{REFERENCES}

[1] Kwok D.Y., Neumann A.W. Contact angle measurement and contact angle interpretation. Advances in Colloid and Interface Science, 1999, 81, 167-249.

[2] Yuehua Yuan and Randall Lee T. Contact Angle and Wetting Properties. In Surface Science Techniques, Springer Series in Surface Sciences; G. Bracco, B. Holst, Eds.; Springer-Verlag, Berlin, Heidelberg, 2013, 51, 3-34; DOI 10.1007/978-3-642-34243-1_1; http://www.springer.com/978-3-642-34242-4. 
[3] Takeuchi M, Sakamoto K, Martra G, Coluccia S, Anpo M. Mechanism of photoinduced superhydrophilicity on the $\mathrm{TiO}_{2}$ photocatalyst surface. J. Phys. Chem. B, 2005, 109(32):15422.

[4] Bredikhin V. I., Burenina V. N., Mamayev Yu. A. and Yashin S. N., Spectral and relaxation properties of the photoconductivity of thin $\mathrm{TiO}_{2}$ films produced by the sol-gel technique. Physical Review \& Research International, 2013, 3(4), 642-665. DOI $: 10.9734 /$ prii/2013/4253.

[5] De Marco C., Eaton S. M., Martinez R. . Solvent vapor treatment controls surface wettability in PMMA femtosecond-laser-ablated microchannels. Microfluid Nanofluid, 2013, 14, 171-176.

[6] Ogilvie I.R.G., Sieben V.J. et al Reduction of surface roughness for optical quality microfluidic devices in PMMA and COC. J. Micromech. Microeng., 2010, 20(6):065016.

[7] B. Ranby, J.F. Rabek. Photodegradation, Photo-Oxidation and Photostabilization of Polymers; Willey-Interscience Publication, London, New York, Sydney, Toronto, 1975.

[8] Bityurin N., Muraviov S., Alexandrov A., Malyshev A. UV laser modifications and etching of polymer films (PMMA) below ablation threshold. Appl. Surf. Sci., 1997, 110, 270-275.

[9] Bityurin N., Afanasiev A., Bredikhin V., Alexandrov A., Agareva N., Pikulin A., Ilyakov I., Shishkin B., and Akhmedzhanov R. Colloidal particle lens arrays-assisted nano-patterning by harmonics of a femtosecond laser. Optics Express 2013, 21 (18), 21485 21490.

[10] Afanasiev Andrei, Bredikhin Vladimir, Pikulin Alexander, Ilyakov Igor, Shishkin Boris, Akhmedzhanov Rinat, Bityurin Nikita. Twocolor beam improvement of the colloidal particle lens array assisted surface nanostructuring. Applied Physics Letters, 2015, 106, $1831021-1831024$.

[11] J.R. Vig, Ultraviolet-ozone cleaning of semiconductor surfaces, research and development, Technical Report SCLET-TR-91-33 (Rev.1), Army Research Laboratory, NJ 07703-5601, U.S.A., 1992.

[12] Matyukhin, S. I. and Frolenkov, K. Yu. Forma kapel zhidkosti, pomeschennykh na tverduyu gorizontalnuyu poverhnost [Shape of liquid drops, placed on a solid horizontal surface]. Kondensirovannyie sredy i mezhfazniye granitsy [Condensed matters and interphase borders, 2013, 15(3), 292-304. (In Russian). 